\title{
ARAC - The Montreal Jewish General Hospital Alzheimer Risk Assessment Clinic
}

\author{
Hyman M. Schipper, Adrienne Liberman, Nora Kelner, Lennie Babins, \\ Lynda Fried, Melanie Bilbul, Rachel Goodman
}

\begin{abstract}
Introduction: In parallel with robust efforts world-wide to develop effective neuroprotection for established disease, resources are being mobilized to delineate risk factors and implement preventive measures in a concerted effort to forestall the anticipated Alzheimer disease (AD) epidemic. A review of heritable and 'acquired' dementia risk factors, many operating at midlife, is presented in a companion paper. Objectives: In 2009, an Alzheimer Risk Assessment Clinic (ARAC) was established at the Jewish General Hospital (Montreal) to address the concerns increasingly being voiced by active middle-aged individuals at risk for AD. A positive family history of $\mathrm{AD}$ and/or perceived changes in personal cognitive function (predominantly short-term memory) are main reasons for referral. The primary objectives of ARAC are to (i) ascertain, inform and mitigate the risks of developing AD in cognitivelyhealthy persons aged 40-65 based on best available medical and epidemiological evidence, (ii) conduct scientific research on midlife dementia risk and prevention in this population and (iii) provide instruction in dementia risk assessment and management to health professionals, clinical/research fellows, medical residents and students. ARAC infrastructure, evaluation protocol, risk profile classification scheme, interventions, knowledge dissemination program, case vignettes, and seminal research projects are described. Conclusions: It is hoped that ARAC and similar initiatives will help prevent or delay dementia by innovating effective interventions based on increasingly nuanced estimation of modifiable AD risk in presymptomatic persons.
\end{abstract}

RÉSUMÉ: La clinique d'évaluation du risque de maladie d'Alzheimer de l'Hôpital général juif de Montréal. Contexte : En parallèle à des efforts soutenus à l'échelle mondiale pour mettre au point une neuroprotection efficace pour traiter la maladie d'Alzheimer, des ressources sont actuellement mobilisées pour définir les facteurs de risque et mettre en place des mesures de prévention par un effort concerté pour contrer l'épidémie de maladie d'Alzheimer (MA) anticipée. Nous présentons, dans un article qui accompagne celui-ci, une revue des facteurs de risque à transmission héréditaire et des facteurs "acquis" de démence, dont plusieurs agissent chez les adultes d'âge moyen. Objectifs : En 2009, une clinique d'évaluation du risque de maladie d'Alzheimer (CERMA) a été établie à l'Hôpital général juif de Montréal pour répondre aux préoccupations exprimées de plus en plus fréquemment par des individus actifs d'âge moyen qui sont à risque de MA. Les principales raisons de consultation sont une histoire familiale positive de MA et/ou des changements perçus de la fonction cognitive personnelle (surtout la mémoire à court terme). Les objectifs primaires de la CERMA sont : 1) d'évaluer, d'informer et de diminuer les risques de présenter la MA chez des individus en bonne santé cognitive qui sont âgés de 40 à 65 ans, en se basant sur les meilleures données médicales et épidémiologiques disponibles ; 2) d'effectuer des recherches scientifiques sur le risque de démence au milieu de la vie et sur la prévention chez cette population et 3) de fournir de l'enseignement sur l'évaluation du risque de démence et sur sa prise en charge aux professionnels de la santé, aux collègues cliniciens ou chercheurs, aux résidents et aux étudiants. Nous décrivons l'infrastructure, le protocole d'évaluation, le plan de classification du profil de risque, les interventions, le programme de diffusion des connaissances, des descriptions de cas et les projets de recherche novateurs de la CERMA. Conclusions : Nous espérons que la CERMA et des initiatives similaires aideront à prévenir ou à retarder la démence au moyen d'interventions efficaces novatrices fondées sur une estimation de plus en plus nuancée des risques modifiables de la MA chez les individus présymptomatiques.

Can. J. Neurol. Sci. 2011; 38: 600-611

\section{Introduction}

Alzheimer disease (AD) is a dementing, neurodegenerative disorder that affects approximately $5-10 \%$ of North Americans over the age of 65 and 30-50\% of those who reach 90 . Many more individuals experience mild cognitive impairment (MCI), a frequent clinical precursor of $\mathrm{AD}$ characterized by cognitive dysfunction (usually involving short-term memory) that fails to meet dementia criteria. It has been estimated that persons with MCI convert to incipient $\mathrm{AD}$ at a rate of approximately $15 \%$ per year, although the speed of progression may vary substantially among subjects ${ }^{1}$. Current pharmacotherapy may transiently improve cognitive function in patients with $\mathrm{AD}$, but no interventions to date have convincingly impacted the underlying

\footnotetext{
From the Centre for Neurotranslational Research, Lady Davis Institute for Medical Research, Jewish General Hospital, Department of Neurology \& Neurosurgery, McGill University, Montreal, Quebec, Canada.

Received November 25, 2010. Final Revisions Submitted March 10, 2011. Correspondence to: Hyman Schipper, Lady Davis Institute, Jewish General Hospital, 3755 Cote St. Catherine Road, Montreal, Quebec, H3T 1E2, Canada.
} 
degenerative process. As described in a companion $\operatorname{article}^{2}$, various medical and psychosocial conditions, many operative at midlife, have emerged as potential risk or protective factors for the development of sporadic AD. Although several of these risk factors are heritable, others are determined by environment or lifestyle and may prove modifiable. Prominent among the latter are: cardiovascular risk factors (hypertension, dyslipidemia, diabetes - which may both exacerbate AD pathology and promote vascular dementia), impoverished mental stimulation, psychological distress (late-life depression, chronic anxiety), and sub-optimal nutrition ('Western' diet) ${ }^{3-7}$. This realization has generated considerable optimism that effective prevention or delay of the disease may soon be possible ${ }^{8-10}$.

\section{A need to know}

The oldest of the 'baby-boomer' generation (née 1946-1964) are now facing retirement and their apprehensions about cognitive deterioration and $\mathrm{AD}$ are increasingly being voiced. As we learn more about individual AD vulnerability profiles - and commensurate with growing general access to 'unfiltered' biomedical information - it is anticipated that an informed public will increasingly inquire about personal estimates of AD risk. Data garnered in the Risk Evaluation and Education for Alzheimer's Disease (REVEAL) study published in $2005^{11}$ and $2009^{12}$ have indicated end-user interest in dementia risk assessment, including predictive apolipoprotein E (APOE) genotyping, even in the perceived absence of effective disease pre-emption. Risk Evaluation and Education for Alzheimer's Disease was a randomized controlled trial designed to determine the impact of $A P O E$ genotype disclosure for $\mathrm{AD}$ risk assessment in adult children of individuals with autopsy-confirmed or clinically-diagnosed AD. The AD risk was ascertained in the control group solely on the basis of family history and gender. Reasons prompting first-degree relatives of AD subjects to seek personal dementia risk evaluations included opportunity to contribute to research $(93.9 \%)$, arrangement of personal affairs $(87.4 \%)$, expectation that effective treatments will be developed $(86.8 \%)$, planning of long-term care $(81.4 \%)$, preparation of family members for the possibility of illness $(77.8 \%)$, expedition of future plans $(75 \%)$, and relief accruing from low-risk assessment $(69.6 \%)^{13,14}$. Speaking to the safety of presymptomatic $A P O E$ testing, middle-aged children of $\mathrm{AD}$ parents who tested negative for the $\varepsilon 4$ allele were relieved to learn of their status and $\varepsilon 4$-positive individuals have thus far shown no stigmata of psychosocial harm after being informed that they were carriers of this risk allele ${ }^{11}$. Moreover, initial evidence suggests that knowledge of personal AD risk profiles may permit individuals to make insightful decisions regarding implementation of potential prevention strategies and spur healthy midlife adults to adopt or modify behaviours that might protect against future disease ${ }^{11,15}$. The motivation to accept 'inconvenient' lifestyle modifications or potentially disagreeable medications may be contingent on the perceived magnitude of the $\mathrm{AD}$ risk $^{11}$.

Some may question the validity of presymptomatic AD risk assessment in the absence of level 1 evidence of effective therapeutic intervention. While the latter would certainly be welcome, this concern should be viewed in the context of the following considerations: (i) Patterson and colleagues ${ }^{5}$ opined, correctly in our perspective, that "very few recommendations can be based on highest quality [level 1] evidence... [because it is] highly unlikely that randomized control trials will ever be conducted on many of the risk factors identified...[therefore] recommendations must necessarily be based on less stringent types of evidence". (ii) Action taken on 'best available', as distinguished from 'best possible', evidence may be warranted in so far as many of the interventions currently implicated in ameliorating $\mathrm{AD}$ risk entail lifestyle adjustments (e.g. dietary modifications, physical exercise, protective headgear) that, in contradistinction to pharmacotherapy, are more likely to prove 'inconvenient' than hazardous ${ }^{10,16}$.

\section{The Alzheimer Risk Assessment Clinic}

Memory Clinics in North America are inundated with referrals for costly and labour-intensive assessments of cognitive decline in our rapidly-aging population and are ill-equipped to address concerns about future cognitive performance raised by healthy midlife persons (the "worried well") at risk for the disease. To meet the clinical needs and conduct essential research involving this midlife population, an Alzheimer Risk Assessment Clinic (ARAC) was established in September 2009 at the Jewish General Hospital (JGH) (McGill University) in Montreal, the first such facility in Canada to our knowledge. The launch of this initiative and its first two years of operation were enabled by generous funding from the Mary Katz Claman Foundation (MKCF), a local non-profit organization affiliated with the Alzheimer Groupe Inc., whose mission is to raise funds and awareness for AD support groups and research in Montreal (http://www.mkc-foundation.com).

\subsection{Objectives}

The primary objectives of ARAC are to (i) ascertain, inform and mitigate the risks of developing $\mathrm{AD}$ in cognitively-intact adults based on best available medical and epidemiological evidence, (ii) conduct scientific research which addresses these concerns in this population and (iii) provide instruction in dementia risk assessment and management to health professionals, clinical/research fellows, medical residents and students. This endeavour may be viewed in the larger context of 'midlife medicine', a relatively recent but burgeoning discipline with considerable clinical and research implications for neurological and 'geriatric' disease ${ }^{17-19}$.

\subsection{Target population and conditions}

The Alzheimer Risk Assessment Clinic was conceived in order to estimate personal dementia risk and provide recommendations for its mitigation in midlife men and women (age 40-65) of all ethnic groups and socioeconomic strata. We opted for an upper age cut-off for ARAC at 65 years as there already exists at our institution a well-established Memory Clinic within the Division of Geriatrics catering to the memory concerns of the elderly. It was felt that the availability of two clinics addressing memory in persons older than 65 at our institution would result in a needless and costly duplication of efforts (already severely strained) as many subjects would likely seek advise at both facilities. The bottom cut-off of 40 years was selected based on recent epidemiological evidence ascribing 
potentially-modifiable susceptibility factors at midlife for latelife dementia and $\mathrm{AD}^{2}$ and a paucity of information about modifiable AD risk at earlier stages of the life cycle. This policy would warrant re-visiting in the event that modifiable AD risk factors determined to be operational prior to midlife become apparent.

Based on earlier experience at the JGH Memory Clinic and private practice (family medicine, neurology, geriatrics, psychiatry), it was anticipated and confirmed that the majority of individuals seeking assessment at ARAC would represent three main categories of concern: 1) Persons with a family history (parents, siblings, etc.) of $\mathrm{AD}$ or other dementia, 2) persons suspecting deterioration of their memory or other cognitive functions, and 3) individuals with known or suspected AD risk factors which are potentially modifiable (e.g. dyslipidemia, diabetes, hypertension). These categories are not mutually exclusive and ARAC patients often manifest various combinations of these factors.

\subsection{ARAC infrastructure and personnel}

The JGH is a 637-bed McGill University teaching hospital and a national leader in the fields of geriatric medicine and aging-related neurodegenerative diseases. It houses the JGH Memory Clinic, an established tertiary care facility for the evaluation of memory loss in Montreal and environs, and the Lady Davis Institute which hosts about a dozen laboratories devoted to basic and clinical research in $\mathrm{AD}$ and related disorders. The Alzheimer Risk Assessment Clinic consists of a reception area and two examination rooms within the hospital's Department of Neurology.

A qualified and enthusiastic team has been assembled to direct and implement ARAC activities at the JGH. The following is a census of the personnel and their primary responsibilities:

- Director: The director designs and oversees ARAC clinical and research activities and performs (or reviews) medical, neuropsychosocial and nutritional client 'intakes', renders diagnostic formulations and proposes interventions (lab tests, referrals, etc.), as detailed in the ARAC Workbook (Appendix 1).

- Research Coordinator: Organizes all aspects of research conducted at ARAC including liaising with JGH Research Ethics Office, recruitment of study volunteers, protocol execution, data entry and analysis, genotyping and public relations.

- Receptionist: Responsible for appointments, referrals, test bookings, correspondence.

- Nurse: Intake of patient demographics, education, occupation, socioeconomic status, vital signs, head circumference $^{20-22}$; blood draws.

- Nutritionist (offsite): ARAC patients with perceived vulnerability in the domain of Nutrition (see below) are referred to a nutritionist at the hospital's Cardiovascular Prevention Centre. The latter obtains a detailed dietary history, including antioxidant/nutraceutical exposure, and makes dietary recommendations.

- Neuropsychologists (two; offsite): In addition to use of standard neuropsychological instruments, novel measurement tools were developed to focus specifically on cognitive and neuropsychiatric profiles germane to the midlife ARAC population (see below). Referred patients receive counseling for enhanced cognitive engagement and/or stress management and neuropsychological data are generated for research projects.

- To fulfill its teaching mandate (see Objectives), ARAC welcomes students, residents and fellows to participate in all aspects of its activities (clinical and research) on a rotating basis. In the first half-year of operation, a neuroscience undergraduate student assisted in the development of the ARAC Workbook, a midlife dementia risk questionnaire and in-house genotyping for apolipoprotein $\mathrm{E}$ and $B D N F$ polymorphisms. The ARAC experience may be particularly attractive to trainees in neurology, neuropsychology, geriatrics, psychiatry and family medicine.

\subsection{ARAC 'flow diagram'}

The following is a bulleted description of the ARAC referral process, sequence of assessments, diagnostic formulation and plan. An ARAC Workbook containing all components of the evaluation is presented as Appendix 1.

- Referral: Individual aged 40-65 with concerns about personal prospects of cognitive decline and dementia is referred to ARAC from a physician's office (generalist or specialist) or a Memory Clinic.

- Nursing Intake: An ARAC nurse interviews/examines the patient and records the following data in a structured and personalized ARAC workbook: (i) demographics, (ii) education, (iii) occupation, (iv) socioeconomic status, (v) vital signs, (vi) height, weight and body-mass index (BMI), and (vii) head circumference.

- Medical Intake: Medical doctors, nurse or trainee obtains or performs (i) family history, (ii) medical history, (iii) surgical history, (iv) list of medications/nutraceuticals, (v) smoking/alcohol history, (vi) leisure physical activity scale, (vii) current laboratory data, (viii) neuropsychosocial screen (neuropsychiatric history, leisure cognitive activity scale, personality scale, Montreal Cognitive Assessment (MoCA) score, social network scale), and (ix) nutritional screen.

- Diagnostic Formulation: On the basis of the above information, the clinic director assigns a Midlife Dementia Risk Score (modified after $^{7}$ ) and ascertains the subject's Dementia Risk Profile: $\mathrm{P} 0=$ no discernible risk; $\mathrm{P} 1=$ Genetic risk (positive family history, apolipoprotein E $\varepsilon 4$ allele when available); P2=Metabolic risk (hypertension, diabetes, metabolic syndrome, low exercise, dyslipidemia, prior stroke, possibly sleep apnea); P3=Nutritional risk (low Mediterranean-style diet score); $\mathrm{P} 4=$ Cognitive risk (low education, low cognitive engagement, head injury); and P5=Psychological risk (chronic anxiety, laterlife depression, impoverished social network). Individuals may harbour risk in no (P0), one or more profile categories (P1-P5).

- Interventions: (i) Unless current laboratory data are available, venous blood is drawn on all subjects for determination of complete blood count (CBC), HgbA1C, electrolytes, glucose, $\mathrm{BUN} / \mathrm{Cr}$, liver functions, albumin, lipid profile, thyroid stimulating hormone (TSH), folate, $\mathrm{B}_{12}$, homocysteine, $\alpha-$ tocopherol and C-reactive protein. (ii) Personal dementia vulnerability profiles and strategies for risk reduction are discussed with patients who, additionally, are provided with educational material on memory fortification and $\mathrm{AD}$ risk prevention (brochures, web links). (iii) Patients are made aware 
of ongoing ARAC research activities (see below) and written informed consent is obtained from willing participants. Informed consent is required for $A P O E$ (and other putative $\mathrm{AD}$ risk allele) genotyping as the latter has been approved by the JGH Research Ethics Committee in the context of 'research' and not for standard clinical practice. This policy is commensurate with current practice guidelines advocating $A P O E$ genotyping in cases of dementia and MCI and in asymptomatic participants in the context of clinical/epidemiological research, but not for prognostication in cognitively-intact persons outside of research. ARAC subjects and their referring physicians are currently not informed of the genetic test results unless the latter are specifically requested. [Recent evidence of a potentially favourable psychosocial impact of $A P O E$ genotype disclosure on asymptomatic midlifers seeking $\mathrm{AD}$ risk assessment may prompt re-consideration of this policy ${ }^{10,16}$. (iv) P2 individuals with hypertension and other cardiac risk factors (and who are not already engaged in a structured cardiovascular fitness program) are referred to the JGH Cardiovascular Prevention Centre. (v) A nutritionist employed at the Cardiovascular Prevention Centre is available to provide dietary advice to ARAC subjects classified as "P3". (vi) Patients falling within the P4 and P5 risk domains are referred offsite to one of two clinical neuropsychologists affiliated with ARAC for further neuropsychological evaluation (see Appendix 2), cognitive exercises and/or stress management. P4 individuals with substantial cognitive deficits (determined by ARAC staff or neuropsychologists) may be scheduled for workup of possible MCI/dementia at the JGH Memory Clinic. (vii) P2 patients with newly-discovered or poorly-controlled diabetes, thyroid dysfunction or dyslipidemia are offered consultation with JGH Endocrinology at the discretion of the referring MD. (viii) Those with suspected sleep apnea are referred to a Sleep Laboratory for definitive diagnosis and therapy. (ix) Genetic counseling with possible testing for gene mutations implicated in familial AD (mutant APP, PS1, PS2) is arranged for P1 subjects with strong family histories of early-onset (< age 60 years) dementia. (x) P1-P5 patients are generally given annual followup appointments in ARAC to re-evaluate ongoing dementia risk and monitor compliance with potential risk-lowering recommendations. Longitudinal follow-up of pre-symptomatic patients with well-defined AD risk factors (including repeated blood-work and, for research subjects, neuroimaging-see below) should provide valuable prospective data concerning the natural history of the disease. (xi) ARAC does not assume the responsibilities for primary medical and psychiatric care of its clientele. For example, if depression is suspected or documented by ARAC personnel and/or affiliated neuropsychologist, consultation with colleagues in the Dept. of Psychiatry is arranged. We do not initiate antidepressant (or for that matter, antihypertensive, antidiabetic or lipid-lowering) therapy in ARAC as such intervention and its monitoring are beyond the mandate, expertise and resources of the clinic and remain the responsibility of the referring physician, the Dept. of Psychiatry, or our institute's Cardiovascular Prevention Centre. Along similar lines, patients are referred to the JGH Memory Clinic for definitive investigation and management of possible $\mathrm{MCI} /$ dementia when significant cognitive impairment is disclosed. By focusing exclusively on dementia risk assessment of a generally healthy and high-functioning population, ARAC is uniquely empowered to satisfy an unmet and growing clinical need with high-throughput and minimal overlap with preexisting medical services and resources.

- Correspondence: Following each ARAC visit, a letter is sent to the referring MD consisting of a brief case report, a summary of the dementia risk profile(s) delineated, an intervention plan, and several salient literature references on $\mathrm{AD}$ risk prevention (see Appendix 3, for sample case summaries).

- Effectiveness assessments: Effectiveness of the interventions will be monitored in two ways: 1) by adducing evidence of improvement in performance or compliance to recommendations over time (e.g annually) on quantitative measures sampled in the ARAC Workbook (vital signs/BMI/HgbA1C, leisure exercise and cognitive scores, Mediterranean diet scores, social network scores, etc.) and, for subjects with cognitive (P4) and psychological (P5) risk factors, formal neuropsychological scores, and 2) externally, in collaboration with Dr. S. Ahmed as described in Section 3.5 (iii).

\subsection{ARAC research}

There currently exists no method which unequivocally arrests, retards or reverses neuronal degeneration and progressive clinical decline in AD and related disorders. While efforts to develop effective neurotherapeutics continue apace, the research community has been galvanized to identify modifiable risk factors and investigate preventive strategies in an attempt to stem the forecasted AD epidemic ${ }^{8,10,23}$. Alzheimer Risk Assessment Clinic is poised to conduct fundamental and translational research along these lines in parallel with its aforementioned clinical activities. Ample opportunity exists for extensive collaboration between ARAC and the highly developed network of clinical facilities and basic laboratories devoted to aging and neurodegeneration at the JGH and affiliated Lady Davis Institute and McGill University. The following is a brief description of several lead projects currently ongoing or in advanced stages of planning at ARAC:

\section{(i) ARAC Neuroimaging Initiative}

While delineation of dementia risk profiles and risk reduction are the raison d'être of ARAC, observational and interventional studies based on dementia outcome per se would entail extensive and costly long-term follow-up periods (20-30 years) and would be exceedingly challenging to perform. In order to circumvent this limitation, the ARAC Neuroimaging Initiative strives to identify surrogate markers of subclinical ("intermediate") phenotypes associated with defined AD risk profiles (P1-P5, vide supra) which may prove useful for monitoring the efficacy of interventional strategies. In collaboration with Dr. A. Thiel (JGH Dept. of Neurology, McGill University), we are evaluating three imaging parameters in ARAC volunteers which are typically related to early neurodegeneration or compensatory plasticity in the normal elderly: (A) Surface cortical thickness (SCT) derived from T1-weighted MR images using a novel method developed in-house ${ }^{24}$, (B) positron emission tomography (PET) with the ${ }^{18} \mathrm{~F}$-labelled benzodiazepine receptor ligand, flumazenil and the new High Resolution Research Tomograph (HRRT) for measurement of in vivo neuronal density ${ }^{24}$, and (C) the PET ligand, ${ }^{11} \mathrm{C}$-PIB (Pittsburgh-B-Compound), a marker of cerebral amyloid deposits. In addition to patients with MCI and 
$\mathrm{AD}$, increased PIB binding has been reported in a proportion of normal elderly subjects possibly reflecting enhanced AD risk. At ARAC, these three imaging modalities are offered in the context of research to midlife subjects at high and low risk for AD representing the various genetic and 'acquired' dementia risk profiles (P1-P5) described above. Molecular testing for the $A P O E \& 4$ allele $^{10}$ and the BDNF Val66Met polymorphism ${ }^{25}$, genetic factors implicated in sporadic $\mathrm{AD}$, is performed on peripheral venous blood leukocytes collected from subjects recruited for neuroimaging (and other consenting ARAC volunteers). We hypothesize that SCT and neuronal density will vary inversely, and PIB labeling will vary directly, with increasing genetic, metabolic, nutritional and/or neuropsychological vulnerability in middle-aged men and women.

\section{(ii) ARAC Biospectroscopy Study}

As alluded to in project (i), the advent of biological markers that correlate with $\mathrm{AD}$ risk profiles at midlife would represent a major breakthrough in the evaluation and management of this chronic neurodegenerative disorder. Biospectroscopy is an optical-based analytical technology that provides a simultaneous and integrated measure of all metabolic activities taking place in a biological sample or field and then uses the accruing spectral data to create a profile of molecular biomarkers. Biospectroscopic analyses in the near-infrared (NIRS) performed in collaboration with Dr. David Burns (McGill Dept. of Chemistry) and Molecular Biometrics Inc. (Norwood, MA) on small volumes of human plasma revealed metabolomic signatures that distinguished, with high sensitivity and specificity, early sporadic AD from normal elderly controls (NEC) and patients with idiopathic Parkinson disease (PD). The metabolomic profiles observed in $\mathrm{AD}$ plasma are consistent with altered oxidation states of protein and other blood-borne constituents $^{26,27}$. Importantly, persons with amnestic MCI exhibited a wide range of values on plasma NIRS, extensively overlapping those of both the AD and NEC cohorts. We conjectured that subjects with plasma NIR spectra in the "AD" range may be at higher risk for imminent deterioration to dementia than neuropsychologically-identical individuals whose spectral signatures fall within "normal" limits. We are set to determine in ARAC volunteers whether pathological NIRS spectra akin to those accruing to MCI/AD occur in midlife individuals at increased risk for $\mathrm{AD}$ (high midlife dementia risk scores; specific risk domains, P1-P5). We will ascertain whether the blood NIRS signatures are impacted by the presence or absence of $A P O E \varepsilon 4$ and BDNF Val66Met polymorphisms and whether pathological NIR spectra correlate with evidence of cortical thinning, neuronal loss and $\beta$-amyloid deposition on neuroimaging (SCT, ${ }^{18} \mathrm{~F}$-flumazenil, and $11 \mathrm{C}$-PIB scanning, respectively) in this population.

\section{(iii) External Evaluation of ARAC}

In light of the increasing burden of chronic illness in Canada, new approaches are emerging to implement innovative models for chronic disease management and prevention. To this end, primary care settings are re-structuring many of their activities in line with key elements of the Chronic Care Model (CCM). The $\mathrm{CCM}$ is an evidence-based client-centered framework that defines the important elements and strategies needed to improve care for individuals with chronic disease ${ }^{28}$. As a new initiative, an external evaluation of ARAC's structure, accessibility, processes, deliverables, knowledge transfer and social impact is highly desirable as a means to ascertain the efficacy of the enterprise and guide it to a maximally successful outcome. In collaboration with Dr. S. Ahmed (McGill School of Physical \& Occupational therapy), an external assessment of ARAC will be conducted using a program evaluation approach, PESTLE (Political, Economic, Social, Technological, and Legal evaluation) instruments and a Long Range Strategic Planning (LRSP) exercise ${ }^{29}$ as part of a national effort to ascertain success factors and barriers to implementation of CCM interventions in Canada $^{30}$.

\subsection{ARAC Knowledge Dissemination Program}

A guiding principle of the ARAC concept is the empowerment of citizens with the knowledge and resources to take matters of $\mathrm{AD}$ risk containment into their own hands ${ }^{28}$. To facilitate this process, multi-media outreach programs complement clinic activities by educating the community at large about key medical and lifestyle factors (diet, exercise, blood pressure control, cognitive engagement, etc.) currently implicated in the management of $\mathrm{AD}$ risk. To help raise this public awareness, patients attending ARAC receive a 20-page public brochure on $\mathrm{AD}$ risks and prevention adapted from a document on this topic provided by the National Institutes of Health, US. In addition, information is made available regarding community-based resources for physical fitness, cognitive engagement, and social stimulation in the greater Montreal area. Institutional and public lectures are presented by ARAC staff, and ARAC activities are disseminated campus-wide via hospital and university periodicals and professional publications. An interactive ARAC website and production of a video documentary on dementia prevention are also under consideration to promote the clinic's educational agenda.

\section{Conclusions}

The Alzheimer Risk Assessment Clinic's mandate is to provide clinical estimates of dementia risk and strategies for its control based on best available medical evidence to midlife individuals seeking such information. The data collected will serve two main purposes: (i) to inform individuals of their personal dementia risk profiles and offer recommendations for their mitigation where possible and (ii) to establish a prospective database on midlife AD risk factors in a large, ethnically-diverse population. The latter should constitute a valuable resource for research on the predispositions and prevention of $\mathrm{AD}$ and other aging-related dementias. It is envisioned that fundamental, translational and clinical research on this defined population will disclose important information concerning the amenability of risk behaviours and co-morbidities to modification, the natural history of cognitive decline as a function of specific midlife risk profiles (Genetic, Metabolic, Nutritional, Cognitive, Psychological) and their interplay, the delineation of surrogate biomarkers (neuroimaging, etc.) of current/future cognitive vulnerability and disease-preventing interventions, and feasibility of this model for the management of other chronic illnesses (e.g. Parkinson disease) in Canada and elsewhere.

With the recent launch of large-scale initiatives like "Prevent Alzheimer's Disease by 2020" in the $\mathrm{US}^{8}$, we may anticipate 
with confidence the rapid accrual of new data linking late-life dementia to potentially-modifiable risk profiles operative in the middle years. It is hoped that initiatives along the lines of ARAC will assist in forestalling the feared AD epidemic by innovating effective prevention based on increasingly nuanced estimation of personal $\mathrm{AD}$ risk in presymptomatic persons.

\section{ACKNOWLEDGMENTS}

The Alzheimer Risk Assessment Clinic is funded by an endowment from the MKCF. Dr. Schipper's laboratory is supported by the Canadian Institutes of Health Research, the MKCF and Molecular Biometrics Inc.

\section{DISCLOSURES}

Hyman Schipper has served as consultant to Osta Biotechnologies, Molecular Biometrics Inc., TEVA Neurosciences and Caprion Pharmaceuticals. He holds stock options in Osta and equity in Molecular Biometrics Inc.

\section{REFERENCES}

1. Chertkow H, Bergman H, Schipper HM, et al. Assessment of suspected dementia. Can J Neurol Sci. 2001;28 Suppl 1:S28-41.

2. Schipper HM, Bilbul M. Risk profiles of Alzheimer disease. Can J Neurol Sci. 2011;38(4):580-92.

3. Hughes TF, Ganguli M. Modifiable midlife risk factors for late-life cognitive impairment and dementia. Curr Psychiatry Rev. 2009;5 (2):73-92.

4. Patterson C, Feightner JW, Garcia A, MacKnight C. General risk factors for dementia: A systematic evidence review. Alzheimer's Dement. 2007:3:341-7.

5. Patterson C, Feightner JW, Garcia A, MacKnight C. Primary prevention of dementia. Alzheimers Dement. 2007;3:348-54.

6. Kivipelto M, Helkala EL, Laakso MP, et al. Midlife vascular risk factors and Alzheimer's disease in later life: longitudinal, population based study. Brit J Med. 2001;322(7300):1447-51

7. Kivipelto M, Ngandu T, Laatikainen T, Winblad B, Soininen H, Tuomilehto J. Risk score for the prediction of dementia risk in 20 years among middle aged people: a longitudinal, populationbased study. Lancet Neurol. 2006;5(9):735-41.

8. Khachaturian ZS, Khachaturian AS. Prevent Alzheimer's disease by 2020: A national strategic goal. Alzheimer's Dement. 2009;5(2): 81-4.

9. Khachaturian ZS, Snyder PJ, Doody R, et al. A roadmap for the prevention of dementia II: Leon Thal Symposium 2008. Alzheimers Dement. 2009;5(2):85-92.

10. Schipper HM. Apolipoprotein E: Implications for AD neurobiology, epidemiology and risk assessment. Neurobiol Aging. 10.1016/j. neurobiolaging.2009.04.021.

11. Roberts JS, Cupples LA, Relkin NR, Whitehouse PJ, Green RC. Genetic risk assessment for adult children of people with Alzheimer's disease: the Risk Evaluation and Education for Alzheimer's Disease (REVEAL) study. J Geriatr Psychiatry Neurol. 2005;18(4):250-5.

12. Green RC, Roberts JS, Cupples LA, et al. Disclosure of APOE genotype for risk of Alzheimer's disease. N Engl J Med. 2009; $361(3): 245-54$
13. Roberts JS. Anticipating response to predictive genetic testing for Alzheimer's disease: a survey of first-degree relatives. Gerontologist. 2000;40(1):43-52.

14. Roberts JS, LaRusse SA, Katzen H, et al. Reasons for seeking genetic susceptibility testing among first-degree relatives of people with Alzheimer disease. Alzheimer Dis Assoc Disord. 2003;17(2):86-93.

15. Fanshawe TR, Prevost AT, Roberts JS, Green RC, Armstrong D, Marteau TM. Explaining behavior change after genetic testing: the problem of collinearity between test results and risk estimates. Genet Test. 2008;12(3):381-6.

16. Schipper HM. Presymptomatic apolipoprotein E genotyping for AD risk assessment and prevention. Alzheimers Dement. [in press] 2011.

17. Finch CE. Toward a biology of middle age. In: Lachman ME, editor. Handbook of midlife development. New York: John Wiley \& Sons. p. 77-108.

18. Lachman ME. Development in midlife. Annu Rev Psychol. 2004; 55:305-31.

19. Severson JA, Marcusson J, Winblad B, Finch CE. Age-correlated loss of dopaminergic binding sites in human basal ganglia. J Neurochem. 1982;39(6):1623-31.

20. Perneczky R, Wagenpfeil S, Lunetta KL, et al. Head circumference, atrophy, and cognition: implications for brain reserve in Alzheimer disease. Neurology. 2010;75(2):137-42.

21. Borenstein Graves A, Mortimer JA, Bowen JD, et al. Head circumference and incident Alzheimer's disease: modification by apolipoprotein E. Neurology. 2001;57(8):1453-60.

22. Schofield PW, Logroscino G, Andrews HF, Albert S, Stern Y. An association between head circumference and Alzheimer's disease in a population-based study of aging and dementia. Neurology. 1997:49(1):30-7.

23. Khachaturian ZS. A roadmap for the prevention of dementia II: Leon Thal Symposium 2008. Alzheimer's Dement. 2009;5 (2):85-92.

24. la Fougère C, Grant $\mathrm{S}$, Kostikov A, et al. Where in-vivo imaging meets cytoarchitectonics: the relationship between cortical thickness and neuronal density measured with high-resolution [18 ${ }^{\mathrm{F}}$ flumazenil-PET. Neuroimage. [In press] 2010.

25. Feher A, Juhasz A, Rimanoczy A, Kalman J, Janka Z. Association between BDNF Val66Met polymorphism and Alzheimer disease, dementia with Lewy bodies, and Pick disease. Alzheimer Dis Assoc Disord. 2009;23(3):224-8.

26. Burns DH, Rosendahl S, Bandilla D, Maes OC, Chertkow HM, Schipper HM. Near-infrared spectroscopy of blood plasma for diagnosis of sporadic Alzheimer's disease. J Alzheimers Dis. 2009; 17:391-7.

27. Schipper HM, Kwok CS, Rosendahl SM, et al. Spectroscopy of human plasma for diagnosis of idiopathic Parkinson disease. Biomarkers Med. 2008;2:229-38.

28. Kreindler S. Lifting the burden of chronic disease: What's worked, what hasn't, what next. Winnipeg Regional Health Authority. 2008.

29. PESTLE Analysis. Renewal Associates, 2003.

30. Ahmed S, Gogovor A, Berman E, et al. Changing healthcare: stakeholder perceptions of the burden of chronic disease and the value of teams, measurements and communication. Healthc Q. 2009;12(2):e1-e13. 


\section{APPENDIX I}

\section{THE ARAC WORKBOOK}

Hôpital général juif

Jewish General Hospital

Name:

JGH U-

\section{Date of assessment:___ $\frac{1}{\text { day } / \mathrm{month} / \text { year }}$}

\section{NURSING INTAKE}

Nurse:

Referring MD/clinic:

Reason for referral:

DEMOGRAPHICS

Date of birth: $\frac{1}{\text { day } / \text { month } / \text { year }}$ Age:

Place of Birth: City / Country

Gender: $\mathrm{F} \quad \mathrm{M}$

Race/Ethnicity: White Black Hispanic Asian Other

Language(s):

Handedness: $\quad \mathrm{R} \quad \mathrm{L}$

Education (years):

Occupation:

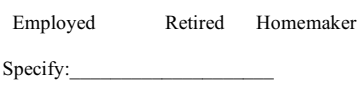

Family income: $<\$ 50,000 \quad 50,000-100,000>100,000-250,000>250,000$

FAMILYMarital status: Single Married Divorced Widowed

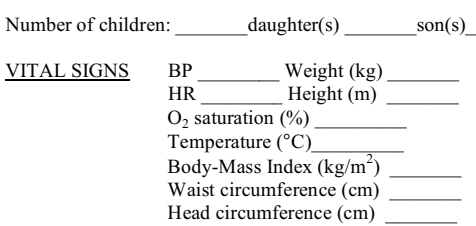

COMMENTS

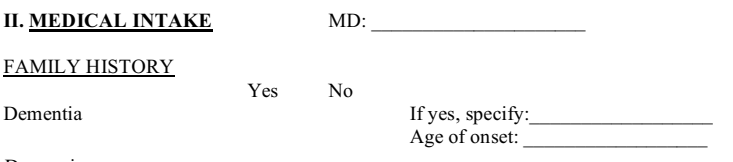

Depression

Other Psychiatric Disease

Down's syndrome

Other Neurological Disease

If yes, specify:

MEDICAL HISTORY

Angina

Myocardial infarct

Heart failure

Arrythmia

Hypertension

Peripheral vascular disease

Diabetes-type 1

Hyperlipidemia

Stroke

Stroke
Cancer

Cancer
CNS infections

Parkinson's disease

Motor neuron disease

Head injury (with loss of consciousness)

Seizure disorder

Thyroid disease

Other metabolic dysfunction

$\mathrm{B}_{12} /$ folic acid deficiency

Blood disorders

Pulmonary disease

Liver disease

Renal disease
SURGERY

General Anesthesia

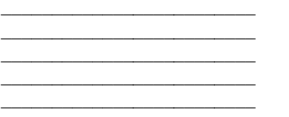

Year

Yes

No

CURRENT MEDICATIONS

Cardiac

Blood Pressure

Anti-inflammatory

Lipid lowering

Blood thinner

Diabetes

Sedative / Hypnotic

Major Tranquilizer

Antidepressant

Cognitive enhancer

Antioxidants

Other

SEX HORMONE EXPOSURE

Oral Contraceptives Estrogen only Progestin only

Combined (E \& P)

Menopause Age

Hormone Replacement Therapy

Estrogen

Progesterone

Androgen (testosterone)

GnRH agonists (Leuprolide, etc.)

ALLERGIES

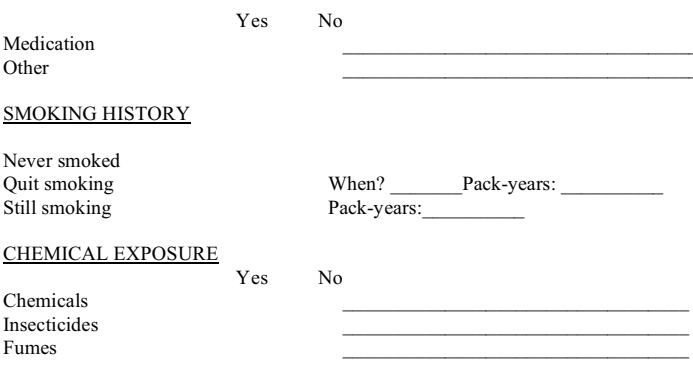

Insecticid

PHYSICAL EXERCISE

Type(s):

Frequency x Duration (Hours/week x years):

LEISURE EXERCISE ACTIVITIES (modified from Verghese et al, 2003)

How often do you participate in the following activities during leisure time?

$\begin{array}{ccccc} & \text { Daily } & 2-6 \text { days/week } & \text { Weekly } & \text { Monthly } \\ \text { Points } & {[6]} & {[5]} & {[4]} & {[3]}\end{array}$

Tennis, etc

Swimming/skiing

Bicycling /jogging

Dancing

Group exercises

Team games

Power walk, golf

Two flights of stairs

Treadmill/stairmaster/Wii

Housework

Leisure Exercise Score: ___ 160

For how long (years) have you been engaged in these activities?

LABORATORY DATA

CBC:

Electrolytes:

Glucose/HgbA1C:

BUN/Cr:

BUN/Cr:
Liver functions:

Lipid profile:

Folate/B12/Homocysteine:

Thyroid :

Iron studies:

ESR/CRP: 
Other:

\begin{tabular}{lll}
\multicolumn{2}{l}{ Neuroimaging: } & \\
\hline GENOTYPING & \\
\hline APOE & Available & Not available \\
BDNF & Available & Not available \\
Tomm40 & Available & Not available
\end{tabular}

Tomm40 Available Not available

III. NEUROPSYCHOSOCIAL SCREEN (NPSS)

NEUROPSYCHIATRIC HISTORY

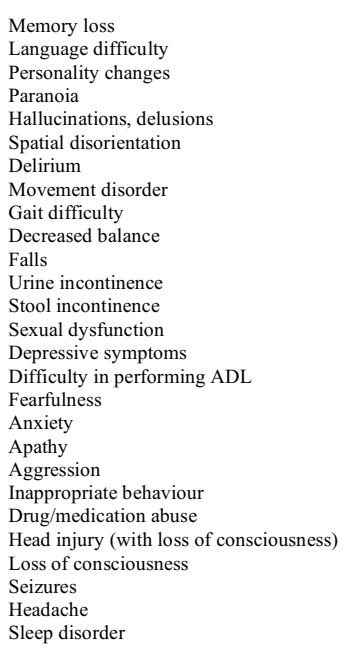

Comment(s)

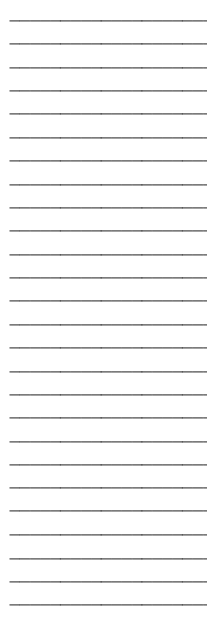

loud snoring

LEISURE COGNITIVE ACTIVITIES (modified from Verghese et al, 2003) How often do you participate in the following activities during leisure time?

$$
\begin{array}{ccccc} 
& \text { Daily } & 2-6 \text { days/week } & \text { Weekly } & \text { Monthly } \\
\text { Points } & {[6]} & {[5]} & {[4]} & {[3]}
\end{array}
$$

Reading books/newspapers Writing for pleasure

Doing puzzles

Playing board games or cards

Organized group discussions

Playing musical instruments

Leisure Cognition Score:

\section{6}

For how long (years) have you been engaged in these activities? How many hours per week do you spend watching television?

\section{PERSONALITY SCALE}

1. ANXIETY

How would you rate your level of anxiety or stress relative to others your age?

1. Far above average or extreme

2. Somewhat above average

3. Average

4. Below average

5. Little or none

\section{DEPRESSION}

How would you rate your level of sadness or depression relative to others your age?

1. Far above average or extreme

2. Somewhat above average

3. Average

4. Below average

5. Little or none

\section{CONTROL}

How much control or influence do you feel you have over events in your life relative to others your age?

1. Little or none

2. Below average

3. Average

4. Somewhat above average

5. Far above average

4. CONSCIENTIOUSNESS

How would you rate your level of conscientiousness or sense of responsibility relative to others your age?

1. Little or none

2. Below average

3. Average

4. Somewhat above average

5. Far above average
5. SPIRITUALITY

How would you rate your level of spirituality relative to others your age?

1. Little or none

2. Below average

3. Average

4. Somewhat above average

5. Far above average

Personality Score:

SOCIAL NETWORK SCALE (LSNS-6/Lubben et al. 2006)

Q1-How many relatives do you see or hear from at least once a month?

$\begin{array}{lccccc}\text { Points } & {[0]} & {[1]} & {[2]} & {[3]} & {[4]} \\ \text { Number of relatives } & 0 & 1 & 2 & 3-4 & 5-8\end{array}$

Q2-How many relatives do you feel at ease with that you can talk about private matters?

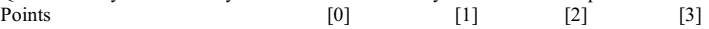

Number of relatives

$\begin{array}{llll}0 & 1 & 2 & 3-4\end{array}$

Q3-How many relatives do you feel close to such that you could call on them for help?

Points

$[0] \quad[1] \quad[2] \quad[3]$

Number of relatives

0

1

2

$3-4$

$5-8$

Q4-How many of your friends do you see or hear from at least once a month?

Points

[0] [1] [2] [3]

Number of friends

0

1

2

3-4

Q5-How many friends do you feel at ease with that you talk about private matters?

Points

[0] [1] [2] [3]

\begin{tabular}{|c|c|c|c|c|c|}
\hline Points & [0] & [1] & [2] & [3] & [4] \\
\hline Number of friends & 0 & 1 & 2 & $3-4$ & $5-8$ \\
\hline
\end{tabular}

Number of friends

0

Q6- How many friends do you feel close to such that you could call on them for help?

Social Network Score

OCA

130

COMMENTS

\section{NUTRITION SCREEN}

MEDITERRANEAN DIET (modified from Trichopoulou et al. 2003)

Meals/week

$$
\text { 7-21 (daily) 4-6 }
$$

$1-3$
(weekly)

$0.25-0.75$

Points [5] [4]

[3]

[2]

Fruits

Vegetables

Nuts

Legumes

Whole cereals

Fish/fish oil

Red Meat

Points

[1]

[2]

[3]

[4]

]

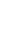

Refined carbohydrate

Saturated fat (cake, fried food)

Mediterranean Diet Score:

ALCOHOL HISTORY

$\begin{array}{lll} & \text { Yes } & \text { No } \\ \text { Alcohol use } & & \text { Type(s): }\end{array}$ \#Drinks/week

ANTIOXIDANT EXPOSURE

Vitamin C (ascorbate)

Vitamin E ( $\alpha$-tocopherol)

Gingko biloba extract

$\beta$-carotene

Other carotenoids (lutein, cryptoxanthine..)

Co-enzyme Q

Idebenone

Lycopene 
Melatonin

Zinc

$\mathrm{N}$-acetylcysteine

Whey protein

$\alpha$-Lipoic acid

Multivitami

Vitamin D

Midlife Dementia Risk Score (MDRS)

[Modified from M. Kivipelto (2006) and M. Sabbagh (2008)]

Name:

Date of Birth:

Date:

Risk

Risk

Female

First-degree relative with Alzheimer's

Age $>65$

Age $>75$

Age $>85$

Education $<7$ years

Head injury with loss of consciousness

Systolic blood pressure $>140 \mathrm{mmHg}$

Diabetes (Type 2)

$\mathrm{BMI}>30$

Cholesterol $>6.5 \mathrm{mmol} / \mathrm{L}$

Low physical activity

Smoking

History of stroke

History of MI

APOE $\varepsilon 4$ positive

$<5=$ LOW, 5-12=MODERATE, $>12=\mathrm{HIGH}$

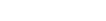

MENTIA RISK PROFILE

$\mathrm{P} 0$ : No or minimal identifiable risks

P1: Genetic (primary relative with dementia, APOE $\varepsilon 4$ )

P2: Metabolic (HTN, DM, hyperlipidemia, metabolic syndrome, MI, CVA, low exercise)

P3: Nutritional (low Mediterranean diet, high Western diet, low antioxidants, alcohol)

P4: Cognitive (low education, low cognitive engagement, brain injury, memory loss)

P5: Psychological (high anxiety, depression; low control, conscientiousness, spirituality)

TESTS

CBC Hgb AlC Electrolytes, Glucose, BUN/Cr, Liver functions, Albumin Fasting Lipid Profile TSH Folate, B12, Homocysteine Iron studies ESR/CRP

Other

\section{INTERVENTIONS}

CV Prevention Centre Nutritional Program Physical exercise program Neuropsych Management

Cognitive engagement
Stress management

Genetic counseling Memory Clinic Endocrinology Sleep Study Head Trauma Prevention

ARAC follow-up 6 Months 1 Year Other

ARAC

RESEARCH

ApoE BDNF Tomm40 Redox profiling MicroRNA Biospectroscopy

Neuroimaging

CT head

MRI

Other

PIB-PET

\section{APPENDIX II}

\section{ARAC Neuropsychological Battery}

ARAC patients displaying potential vulnerabilities for future dementia in the domains of Cognitive (P4) or Psychological (P5) are referred to neuropsychologists employed by the clinic for indepth neuropsychological evaluation and management (memorystrengthening exercises, stress reduction, etc.). A neuropsychological evaluation is a systematic examination of the relationship between brain-function and how a person thinks, feels, and acts. A comprehensive assessment focuses on these brainbehavior relationships and the impact of brain disease and/or insult on the cognitive, sensorimotor, emotional, and adaptive abilities of the individual. Patients referred from ARAC undergo investigation of intellectual ability, personality and mood, memory and learning, language, praxis, visuo-spatial skills, perceptual and motor function. They are also subjected to tests of executive function comprising attention/concentration, mental flexibility, sequencing, judgment, abstract reasoning, problems solving, planning, and organization.

To accomplish the latter, the following neuropsychological instruments and test batteries were carefully selected for optimal evaluation of healthy midlife individuals within the span of a single 2.5 hour visit:

(i) Kaufmann Brief Intelligence Test 2nd Ed. (KBIT2)

[Alan S. Kaufmann, Nadeen L. Kaufmann (2004) Kaufmann Brief Intelligence Test 2nd. Ed. Manual. Pearson, Inc.] The KBIT2 is a brief, individually based measure of verbal/non-verbal intelligence, spanning the ages of 4 to 90 years. The test takes approximately 15 to 30 minutes to administer and yields three scores: Verbal, Nonverbal, and the Overall score, known as the IQ Composite. From a theoretical perspective, the Verbal subtests measure crystallized ability and the Nonverbal subtest measures fluid reasoning. The KBIT2 is useful for measuring the intelligence of diverse groups for research purposes. The Verbal score comprises two subtests (Verbal Knowledge and Riddles) and measures Verbal skills by assessing the person's verbal knowledge, range of general information, verbal concept formation, and reasoning ability. The Verbal Knowledge subtest is a 60 -item measure of receptive vocabulary and range of general information about the world (nature, geography, the arts, science, etc). The Riddles subtest has 48 items that measure verbal comprehension, reasoning, and vocabulary knowledge. The Nonverbal score (the Matrices subtest) measures the ability to solve new problems by assessing an individual's ability to perceive relationships and complete visual analogies through pictures and abstract designs. Matrices is a 46item nonverbal measure composed of several types of items involving visual stimuli, both meaningful (people and objects) and abstract (designs and symbols). Internal consistency reliabilities for the Verbal score range from .86 to .96 (mean=.91). For the Nonverbal scores reliabilities range from .78 to .93 (mean=.88). The reliability of the IQ Composite is excellent, ranging from .89 to .96 and averaging .93 across the entire age range. Test-retest reliabilities of the Verbal score are high, ranging from .88 to .93 (mean=.91), and those of the Nonverbal score are good, ranging from .76 to .89 (mean=.83). Test-retest reliabilities of the IQ Composite, ranging from .88 to. 92 (mean=.90) is excellent.

(ii) Hopkins Verbal Test Revised (HVLT-R)

[Jason Brandt, Ralph HB Benedict (2001). Hopkins Verbal Test Revised. Manual. Odessa, Fla: Psychological Assessment Resources] The HVLT-R is used to provide a brief assessment of verbal learning and memory. It consists of a list of 12 nouns with four words drawn from each of the three semantic categories (e.g., four-legged animals, precious stones, and human dwellings). The HVLT-R includes three learning trials, a 20-25 minute delayed recall trial immediately followed by a yes/no recognition trial 
selecting between 12 target and 12 nontarget words. About 15 minutes is required for the test, excluding the delay interval. Reliability coefficients for the four primary HVLT-R variables were .74 for Total Recall, .66 for Delayed Recall, .39 for \% Retention, and .40 for the Recognition Discrimination Index.

\section{(iii) Brief Visuospatial Memory Test Revised (BVMT-R)}

[Ralph HB Benedict (1997). Brief Visuospatial Memory Test Revised. Manual. Odessa, FLA: Psychological Assessment Resources] The BVMT-R measures visual learning and memory using a three-trial learning paradigm. The test consists of immediate recall, rate of acquisition or learning, delayed recall, and recognition. The person is shown a matrix containing six simple geometric visual designed in a $2 \times 3$ matrix presented for 10 seconds each of the three times. After each exposure, the individual is asked to reproduce as many of the designs as possible, in the same location as they appear on the display. After a 25-minute delay the person is asked to reproduce the designs again followed by a recognition trial in which the individual is shown 12 designs one at a time. The subject is asked to respond "yes" to those designs that were included in the original matrix and "no" to distracters. The manual provides a scoring guide for each design according to drawing and spatial placement criteria and various numerical scores are obtained. Reliability coefficients range from .60 for trial 1 to .84 for trial 3 . The reliability coefficient for the total recall is .80 .

(iv) Color Trails Test (CTT)

[Louis F. D'Elia, Paul Satz, Craig Lyons Uchiyama, Travis White. (1996). Color Trails Test. Manual. Odessa, Fla: Psychological Assessment Resources] The CTT measures speed of attention, sequencing, mental flexibility, visual search, and motor function. The adult version is for individuals age 18 to 89 . The CTT has two parts and is designed to minimize the influence of language so it could be used in cross-cultural settings. In Trial 1 the respondent is instructed to rapidly draw a line connecting the circles numbered 1 through 25 in consecutive order. Circles with odd numbers are pink and circles with even numbers are yellow. The incidental fact that color alternates with each succeeding number is not mentioned. For Trial 2 the examiner instructs the respondent to rapidly draw a line between numbered circles, maintaining the sequence of numbers, but alternating between pink and yellow colors. The time for the completion of each part 1 and 2 is recorded in seconds. In addition, this test includes a qualitative scoring of number of errors, nearmisses, and prompts; further, an "Interference Index" is calculated. Both Parts 1 and 2 require perceptual tracking of a sequence and speeded performance. However, Part 2 also requires divided attention. The Interference Index is an indicator of the need to elucidate the added task requirements of Part 2 and is thought to be a pure measure of the interference, attributable to the more complex divided attention and the alternating sequencing tasks in Part 2. Several CTT scores present problems for computing traditional correlation coefficients between the first and second testing. The ranges of the errors, prompts, near-misses, and interference index scores are restricted due to the infrequency with which normal subjects demonstrate these performance features. This artificially reduces the magnitude of the reliability coefficients; thus, it was decided that test-retest reliabilities will be considered only for CTT variable with sufficient range and distribution scores, such as completion time. For the CTT, two-week reliability is reported as marginal, .64, for Part 1 and acceptable to high, .79, for Part 2.

(v) The Clock Drawing Test

[Rouleau I, Salmon DP, Butters N. (1996). Longitudinal Analysis of Clock Drawing in Alzheimer's disease patients. Brain and Cognition, 18, p. 79-87] No specific test materials are required. The examinee is requested to generate a freehand drawing of a clockface including all the numbers. The person is then prompted to indicate the time "11:10". A 10-point system for scoring is used with 2 points for clock-face integrity, 4 points for the presence and sequencing of the numbers, and 4 points for the presence and placement of the hands. The overall correlation between the total scores (three judges) obtained with Sunderland, T, et al, (1989)'s rating scale and with this revised 10-point Rouleau scale was .89.

(vi) Boston Naming Test (BNT)

[Kaplan EF, Goodglass H, Weintraub S. (1983). The Boston naming Test. Philadelphia: Lippincott Williams \& Wilkins] BNT assesses visual naming ability using black and white drawings of common objects. Four 15-item versions have been developed out of the original 60 -item test. Internal consistency for the 60 -item form ranges between .78 and .96 .

\section{(vii) Beck Depression Inventory, 2nd Edition (BECK-II)}

[Aaron Beck, Steer RA. (1987). Beck Depression Inventory. Manual. San Antonio, Texas: Psychological Corporation] The Beck-II is a 21-item self-report instrument for measuring the presence and severity of depression. Each of the 21 BDI-II items requires the respondent to endorse one of four options scored 0 to 3 , with increasing scores reflecting greater severity of a depressive symptom. Item-total correlations range from .35 to .68 . Over short intervals, test-retest correlations are adequate (.74 to .75$)$ to high (.93 to .96$)$.

(viii) Verbal Fluency Test D-KEFS

[Delis DC, Kaplan E, Kramer JH. (2001). Delis-Kaplan Executive Function System. Manual. San Antonio, Texas: Psychological Corporation]. The examinee is asked to say words that begin with a specified letter (B-H-R). Proper names and numbers are not allowed and the time allotted for each letter is 60 seconds. This task evaluates fluency of lexical item generation and requires selfmonitoring of responses. The internal consistency for Letter Fluency Total Score is high $(.80-.89)$.

(ix) NEO Personality Inventory-Revised (NEO PI-R ${ }^{T M}$ )

[Paul T. Costa, Jr, Robert R. McCrae, Odessa, Fla: Psychological Assessment Resources] The NEO PI-R, the standard questionnaire measure of the Five Factor Model (FFM), provides a systematic assessment of emotional, interpersonal, experiential, attitudinal, and motivational styles. The NEO PI-R is a concise measure of the five major domains of personality, as well as the six traits or facets that define each domain. Together, the five domain scales, viz., Neuroticism, Extraversion, Openness, and 30 facet scales of the NEO PI-R including the scales for the Agreeableness and the Conscientiousness domains, facilitate a comprehensive and detailed assessment of normal adult personality. This instrument is appropriate for individuals aged 17 to 89 and can be administered in 30-40 minutes. Internal consistency coefficients for the two available Forms, $\mathrm{R}$ and $\mathrm{S}$, ranges from .86 to .95 for domain scales and from .56 to .90 for facet scales. 


\section{APPENDIX III}

\section{Case Vignettes}

\section{Patient D.B}

D. B. is a 62-year-old man who was concerned about his risk of developing $\mathrm{AD}$ because he has a first degree relative (mother) with the disease. He has not noticed changes in his memory or other cognitive functions. ARAC evaluation revealed vulnerabilities for $\mathrm{AD}$ in the following dementia risk domains*: P1 (Genetic): mother developed dementia (likely AD) at age 75. P2 (Metabolic): hypertension (BP 158/94), body-mass index (BMI) 34.5, current smoking (67 pack-year), insufficient exercise (13/60 leisure exercise score), possible sleep apnea. P3 (Nutritional): Mediterranean diet score (33/50). P4 (Cognitive): Low leisure cognitive engagement (11/36 leisure cognition score). No apparent risks were delineated in domain P5 (Psychological). His overall Midlife Dementia Risk Score (MDRS) was 11.5 placing him in a moderate to high risk category*. Intervention: 1) Blood tests: CBC, electrolytes, liver functions, fasting lipid profile, $\mathrm{TSH}$, folate, $\mathrm{B}_{12}$, homocysteine, $\alpha$-tocopherol. 2) Referral to Cardiovascular Prevention Centre: blood pressure control, exercise program. 3) Referral to Nutritionist: weight reduction, Mediterranean diet. 4) Neuropsychology assessment: cognitive engagement. 5) Sleep Study [confirmed obstructive sleep apnea]. 6) Return to ARAC annually (until age 65) to re-evaluate Alzheimer risk status and compliance with recommended interventions.

\section{Patient L. V.}

L. V. is a 51-year-old woman who was concerned about her risk of developing AD because she has a first degree relative (mother) with memory loss. The patient has noticed no significant changes in her own memory or other cognitive functions. Evaluation revealed no vulnerabilities in any of the major risk domains (P1-P5). Her mother has mild short-term memory loss but does not, by history, meet clinical dementia criteria. Her MDRS is 1.5 (low) and her dementia risk profile is $\mathrm{P} 0$ (no or minimal identifiable risks). Interventions: 1) No referrals or ARAC follow-up indicated. 2) Excellent 'control' (P0) subject for ARAC research studies (see Section 3.5). 3) Her mother may benefit from assessment in Memory Clinic.

\section{Patient B. S.}

B.S. is a 64-year-old woman who was worried about her risk of developing $\mathrm{AD}$ because she has a first degree relative (father) with dementia and has noticed changes in her short-term memory. There were no other cognitive or psychological symptoms. Evaluation revealed vulnerabilities for $\mathrm{AD}$ in the following risk domains: $\mathrm{P} 1$ (Genetic): Father developed dementia (vascular or mixed) at age 93. P4(Cognitive): MoCA score 25/30. No apparent risks discerned in the domains: P2(Metabolic), P3 (Nutritional), P5 (Psychological). Her MDRS score was 4.5 (low). Interventions: 1) Blood tests. 2) Neuropsychology assessment: cognitive engagement. 3) Return to ARAC in one year. 4) Referral to Memory Clinic if memory deficits progress or other cognitive symptoms develop.

\section{Patient A. V.}

A. V. is a 56-year-old woman who is concerned about her risk of developing $\mathrm{AD}$ because she has first degree relatives with the disease. She denies changes in her own memory or other cognitive functions. Evaluation revealed vulnerabilities in the following risk domains: P1 (Genetic): dementia diagnosed in mother at age 62, maternal uncle age 58, and mother's paternal cousin age 70. P2 (Metabolic): low leisure physical activity score (24/60). P4 (Cognitive): low leisure cognitive engagement score (12/36). P5 (Psychological): high anxiety, dysphoria, low control, limited social network (11/30 on Social Network Scale). No apparent risks were noted in domain P3 (Nutritional). MoCA score $=28 / 30$. Interventions: 1) Blood tests. 2) Encourage leisure-time exercise. 3) Neuropsychological evaluation: cognitive engagement and stress management. 4) Genetic Counseling for possible familial Alzheimer disease [testing already in progress in Italy]. 5) Return to ARAC annually (until age 65).

\section{Patient J. W.}

$\mathrm{J}$. W. is a 63-year-old man who was concerned about his risk of developing AD because he had noticed changes in his short-term memory. Evaluation revealed vulnerabilities in the following risk domains: P2 (Metabolic): myocardial infarct at age 45, diabetes mellitus diagnosed at age 61. P4 (Cognitive): memory deficits, low cognitive engagement (leisure cognitive activity scale 16/36). There were no overt vulnerabilities in the risk domains P1 (Genetic), P2 (Nutritional) or P5 (Psychological). His MDRS was 5.5 (lowmoderate). Interventions: 1) Blood tests. 2) Neuropsychological evaluation: cognitive engagement. 3) ARAC follow-up in one year.

6. Patient V.S

V. S. is a 51-year-old woman who was worried about her risk of developing $\mathrm{AD}$ because her mother developed the disease around age 70 . The patient noticed no significant changes in her own memory or other cognitive functions. Evaluation disclosed vulnerabilities in two AD risk domains: P1 (Genetic): primary relative (mother) with late-onset $\mathrm{AD}$. P2 (Metabolic) low leisure time physical activity (score 26/60). There were no overt vulnerabilities in the risk domains P3 (Nutritional), P4 (Cognitive) or P5 (Psychological). Her MoCA score was 30/30 and her was 6.7 (moderate). Interventions: 1) Blood tests. 2) Encourage compliance with the physical fitness program she had registered for. 3) Her current medical status, diet and level of cognitive engagement are favourable and should be maintained. 4) Return to ARAC annually (until age 65).

\section{Patient P. B}

P.B. is a 63-year-old woman who was concerned about her risk of developing AD because she has a first degree relative (mother) with dementia. Over the last two years, she has noticed mild changes in her own short-term memory and some word-finding difficulties. ARAC evaluation revealed vulnerabilities in the following risk domains: $\mathrm{P} 1$ (Genetic): Mother diagnosed with $\mathrm{AD}$ at age 70 ; mother's niece with dementia in her early 70s. P2 (Metabolic): type 2 diabetes, low exercise (leisure exercise score 18/60). P5(Psychological): chronic high anxiety. No apparent risks were noted in the domains P3 (Nutritional) and P4 (Cognitive) despite her cognitive symptoms (MoCA score 30/30). Her MDRS was 11.6 (moderate-high). Interventions: 1) Blood tests. 2) Referral to JGH Cardiovascular Prevention Centre. 3) Referral for neuropsychological evaluation: stress management. 4) Follow-up ARAC visit in one year.

\section{Patient L. T.}

L. T. is a 47 year-old woman who is concerned about her risk of developing $\mathrm{AD}$ because she has first and second degree relatives with dementia. She had not noticed changes in her own memory. Evaluation revealed vulnerabilities in the following risk domains: $P$ 1 (Genetic): father diagnosed with $\mathrm{AD}$ at age 66; six of 10 of father's siblings and two maternal aunts have/had dementia. P2 (Metabolic): Dyslipidemia and low exercise (leisure exercise score 29/60). No apparent risks in domains P3 (Nutritional), P4 (Cognitive), and P5 (Psychological). Her MoCA score was 29/30 and her MDRS 8.3 (moderate). Interventions: 1) Blood tests. 2) JGH Cardiovascular Prevention Centre. 3) Annual ARAC followup.

9. Patient S.M.

S.M. is 54 year-old woman who was concerned about her risk of developing AD because she has become more forgetful. Over the last two years, she has noticed mild changes in her short-term memory, often misplacing household objects, and some wordfinding difficulties. Our evaluation revealed vulnerabilities in the following risk domains: P2 (Metabolic): hypertension, low exercise (leisure exercise score $=19 / 60)$, BMI 31.7. P3 (Nutritional): 
Mediterranean diet score $=31 / 50$. P4 (Cognitive): Leisure cognitive activities score 16/36); MoCA = 27/30. P5 (Psychological): high anxiety, fearfulness, post-menopausal aggression. Minimal risk was delineated in domain P1 (Genetic): maternal grandfather with dementia onset age 85. Interventions: 1) Blood tests. 2) JGH Cardiovascular Prevention Centre. 3) Nutritionist. 4) Neuropsychology evaluation: cognitive engagement, stress management. 5) Annual ARAC follow-up.

10. Patient B. P.

B. P. is a 62 year-old woman who is concerned about her risk of developing $\mathrm{AD}$ because she has become more forgetful and has a family history of dementia. Over the last 12 months, she has noticed changes in her short-term memory, often misplacing household objects and forgetting recent events. Evaluation revealed vulnerabilities in the following risk domains: P1 (Genetic): father with frontotemporal dementia onset age 75 [implications vis-à-vis $\mathrm{AD}$ risk for B.P. unclear]. P5 (Psychological): high anxiety and dysphoria. Minimal risk was delineated in the domains P2 (Metabolic), P3 (Nutritional) and, despite her symptoms, P4 (Cognitive). Her MoCA score was 28/30. Interventions: 1) Blood tests. 2) Neuropsychology evaluation: stress management. 3) Annual ARAC follow-up.

\section{Patient K. R.}

$\mathrm{K}$. R. is a 46 year-old woman who is concerned about her risk of developing $\mathrm{AD}$ because she has become more forgetful. She complains of both short- and long-term memory loss of about one year's duration. Evaluation revealed vulnerabilities in the following risk domains: P4 (Cognitive): head trauma with loss of consciousness age 15; leisure cognitive activities score 13/36; MoCA score 29/30. P5 (Psychological): depression, mood lability, low social support network (social network score $=9 / 30$ ). Minimal or no risk was delineated in the domains P1 (Genetic), P2 (Metabolic) and P3 (Nutritional). Interventions: 1) Blood tests. 2) Neuropsychology evaluation: cognitive engagement and stress management. 3) Annual ARAC follow-up.

*[Individual dementia risk profiles and MDRS may be modified following initial ARAC evaluation pending results of APOE (presence of $\varepsilon 4$ allele) and $\mathrm{BDNF}$ (val/met polymorphism) genotyping]. 